\title{
Secure Transmission Design for Cooperative NOMA in the Presence of Internal Eavesdropping
}

\author{
Binbin Su, Wenjuan Yu, Member, IEEE, Hongbo Liu, Arsenia Chorti, Senior Member, IEEE \\ and H. Vincent Poor, Life Fellow, IEEE
}

\begin{abstract}
The application of successive interference cancellation (SIC) introduces critical security risks to cooperative non-orthogonal multiple access (NOMA) systems in the presence of untrustworthy network nodes, referred to as internal eavesdroppers. To address this potential security and reliability flaw, by assuming all users are untrusted, this letter investigates the effective secrecy throughput (EST) for a cooperative NOMA system, where a near user serves as an amplify-and-forward relay to help forward the information of a far user. Considering the inverse power allocation and SIC decoding order, a novel jamming strategy is proposed to enhance the security performance of the far user. Gauss-Chebyshev approximations of ESTs over Nakagami- $m$ channels are derived. Asymptotic EST expressions are proposed to provide further insights. Numerical results demonstrate that the proposed jamming strategy and the inverse power allocation and SIC decoding order are both essential for achieving positive secrecy rates for both users.
\end{abstract}

Index Terms-Non-orthogonal multiple access, untrusted internal users, effective secrecy throughput

\section{INTRODUCTION}

Non-orthogonal multiple access (NOMA) has drawn significant attention due to its potential to improve spectral efficiency by serving multiple users over a single resource block [1]. Moreover, cooperative NOMA is regarded as an effective way to guarantee fairness among users, where a near NOMA user acts as a relay to help establish connections between the base station (BS) and far weak users [2]. However, due to the broadcast nature of wireless communication networks as well as the application of successive interference cancellation (SIC), NOMA is faced with severe security concerns [3], [4]. The security performance of cooperative NOMA with external eavesdropping has been studied in [5]-[7]. Furthermore, as network nodes of NOMA systems may be untrusted, NOMA systems are exposed to risks of internal eavesdropping. Secure transmission for non-cooperative NOMA systems with internal eavesdropping has been investigated in [8]-[10].

Note that the above studies either consider cooperative NOMA with external eavesdropping [5]-[7], or non-cooperative with only one untrusted user [8]-[10]. For cooperative NOMA systems, since SIC can be applied at all users [8], on one

Binbin Su and Hongbo Liu are with the National Key Laboratory of Science and Technology on Vessel Integrated Power System, Naval University of Engineering, Wuhan, 430033, China (email: subinbinbin_sdu@yahoo.com, myspace218@163.com).

Wenjuan $\mathrm{Yu}$ is with the School of Computing and Communications, Lancaster University, Lancaster LA1 4YW, U.K. (email: w.yu8@lancaster.ac.uk).

Arsenia Chorti is with ETIS, UMR 8051, CY University, ENSEA, CNRS, France (email: arsenia.chorti@ensea.fr). Arsenia Chorti has been supported by the eNiGMA and PHEBE projects of the CYU Initiative of Excellence and the CNRS IEA project PEGASUS.

H. Vincent Poor is with the Department of Electrical and Computer Engineering, Princeton University, Princeton NJ 08544 USA. (email: poor@princeton.edu). hand, it is difficult to achieve positive secrecy rates for a far user since an untrusted near user can easily intercept messages intended for the former due to the stronger channel gain between the BS and the near user [11]. On the other hand, an untrusted far user may access the information of a near user, which results in confidential information leakage. Therefore, for cooperative NOMA in the presence of internal eavesdropping, both near user and far user are faced with risks of being eavesdropped upon, and it is of importance to study security issues for cooperative NOMA with untrusted users.

We have, however, noticed that the effective secrecy throughput (EST), a measure of the rate at which information can be transferred securely in the presence of eavesdroppers [12], has not been studied for cooperative NOMA with untrusted internal adversaries. In this letter, we propose an approach to secure the transmissions in a two-user cooperative NOMA, using EST as the performance metric ${ }^{1}$, for situations in which a near user $U_{1}$ plays the role of an amplify-andforward (AF) relay for a far user $U_{2}$. Different from [8]-[10], we consider a purely antagonistic network in which both $U_{1}$ and $U_{2}$ are untrusted, and can act as passive eavesdroppers intercepting confidential messages intended for the other user. More importantly, we consider the inverse power allocation and SIC decoding order of standard NOMA, i.e., more transmit power is allocated to $U_{1}$, so that $U_{1}$ should first decode its own message. It is noted that though less power is allocated to $U_{2}$, the reliability is still guaranteed in our model as EST ensures that the achievable data rate is larger than or equal to the rate requirement. To enhance security performance, a novel jamming strategy is proposed, where the jamming signal is sent by $U_{2}$ to confuse $U_{1}$. Compared with external jamming [10], [13], the proposed strategy avoids communication overhead, and the jamming can be eliminated by $U_{2}$ before decoding its own information.

In this letter, we show that jamming and inverting power allocation and SIC decoding order are both essential to achieve positive ESTs for both NOMA users. If we allocate more power to $U_{2}$, according to the standard NOMA, its message $x_{2}$ will be decodable at $U_{1}$ and thus is unprotected. If jamming is used to induce secrecy for $x_{2}$ leaving $U_{1}$ unable to decode $x_{2}$, then $U_{1}$ will not be able to decode its own message $x_{1}$ either (because of the SIC order). To validate the performance of the proposed strategy, Gauss-Chebyshev approximations are derived for the ESTs. Numerical results demonstrate that our jamming strategy outperforms the benchmark scheme and ensures positive secrecy rates for both users.

\footnotetext{
${ }^{1}$ The considered two-user model can be easily extended to multiple NOMA clusters by performing user pairing, where each cluster is composed of a near user and a far user. By allocating orthogonal frequency bands to different NOMA clusters, each NOMA pair can be managed independently.
} 


\section{System Model}

Consider a cooperative NOMA system, which is composed of one BS and two users, i.e., a near user $U_{1}$ and a far user $U_{2}$. The BS transmits information to $U_{2}$ with the help of $U_{1}$, since there is no direct link between the BS and $U_{2}$ due to shadow fading and severe blocking. $U_{1}$ and $U_{2}$ are considered to be untrusted by each other, i.e., both $U_{1}$ and $U_{2}$ can act as internal eavesdroppers. Each node is equipped with a single antenna and operates in half-duplex mode. The channel coefficients between the BS and $U_{1}$, and from $U_{1}$ to $U_{2}$ are denoted as $h_{1}$ and $h_{12}$, respectively. All channels are assumed to experience Nakagami- $m$ fading with $E\left[\left|h_{i}\right|^{2}\right]=\Omega_{i}$ and fading parameters $m_{i}, i=\{1,12\}$.

During the first slot, the BS transmits the superimposed signals of $x_{1}$ and $x_{2}$ to the near user. Following Wyner's work on secrecy capacity [14], [15], $x_{1}$ and $x_{2}$ are coded as the code-word rates $\left(R_{1, t}, R_{2, t}\right)$ and secrecy rates $\left(R_{1, s}, R_{2, s}\right)$, respectively. Meanwhile, the far user transmits a jamming signal. The received signal at $U_{1}$ can be modeled as

$$
y_{1}=h_{1}\left(\sqrt{a_{1} P_{s}} x_{1}+\sqrt{a_{2} P_{s}} x_{2}\right)+\sqrt{P_{I}} x_{0}+n_{1},
$$

where $P_{s}$ is the transmit power at the BS, $a_{1}$ and $a_{2}$ are the power coefficients of $U_{1}$ and $U_{2}$ with $a_{1}+a_{2}=1$ and $a_{1}>a_{2}$, $n_{1}$ denotes additive white Gaussian noise (AWGN) at $U_{1}$ with variance $\sigma^{2}, x_{0}$ is the jamming signal and $P_{I}$ denotes the received jamming power at $U_{1}$.

Since more power is allocated to $x_{1}$, based on the principle of SIC, $U_{1}$ first decodes $x_{1}$ by treating $x_{2}$ as interference, and then $U_{1}$ may eavesdrop upon the signal of $U_{2}$, i.e., $U_{1}$ decodes $x_{2}$ after subtracting the decoded $x_{1}$. Therefore, the received signal-to-interference-ratio (SINR) of $x_{1}$ and the eavesdropped SINR of $x_{2}$ at $U_{1}$ can be expressed as

$$
\begin{gathered}
\gamma_{1}=\frac{a_{1} \rho_{s}\left|h_{1}\right|^{2}}{a_{2} \rho_{s}\left|h_{1}\right|^{2}+\rho_{I}+1}, \\
\gamma_{1 \rightarrow 2}=\frac{a_{2} \rho_{s}\left|h_{1}\right|^{2}}{\rho_{I}+1},
\end{gathered}
$$

where $\rho_{s}=\frac{P_{s}}{\sigma^{2}}$ and $\rho_{I}=\frac{P_{I}}{\sigma^{2}}$.

During the second transmission slot, $U_{1}$ amplifies and forwards its received signal to $U_{2}$ with an amplifying coefficient $G$. So, the received signal at $U_{2}$ can be expressed as

$$
y_{2}=h_{12} y_{1} G+n_{2} \text {. }
$$

Here, $G=\sqrt{\frac{P_{R}}{P_{s}\left|h_{1}\right|^{2}+P_{I}+\sigma^{2}}}$, where $P_{R}$ denotes the transmit power at $U_{1}$, and $n_{2}$ is AWGN at $U_{2}$ with variance $\sigma^{2}$.

As $x_{1}$ is allocated with more power, $x_{1}$ is decoded by treating the signal of $x_{2}$ as noise. Consequently, at $U_{2}$, the received SINR to intercept the message intended for $U_{1}$ can be expressed as

$$
\gamma_{2 \rightarrow 1}=\frac{a_{1} \rho_{s} \rho_{R}\left|h_{1}\right|^{2}\left|h_{12}\right|^{2}}{a_{2} \rho_{s} \rho_{R}\left|h_{1}\right|^{2}\left|h_{12}\right|^{2}+\rho_{s}\left|h_{1}\right|^{2}+\rho_{R}\left|h_{12}\right|^{2}+\rho_{I}+1},
$$

where $\rho_{R}=\frac{P_{R}}{\sigma^{2}}$.

After subtracting the information of $U_{1}$ from the received signals, the received SINR of $U_{2}$ is given by

$$
\gamma_{2}=\frac{a_{2} \rho_{s} \rho_{R}\left|h_{1}\right|^{2}\left|h_{12}\right|^{2}}{\rho_{s}\left|h_{1}\right|^{2}+\rho_{R}\left|h_{12}\right|^{2}+\rho_{I}+1} .
$$

\section{Secrecy Performance Analysis}

In this section, EST is adopted as the performance metric. EST is defined as the average transmitted secrecy rate from transmitter to receiver without being successfully eavesdropped upon, which characterizes the reliability and security of the system.

\section{A. Exact Expressions}

The ESTs of $U_{1}$ and $U_{2}$ are defined as

$$
\begin{aligned}
& \eta_{1}=R_{1, s} \operatorname{Pr}\left[\gamma_{1}>\theta_{1, t}, \gamma_{2 \rightarrow 1}<\theta_{1, s}\right], \\
& \eta_{2}=R_{2, s} \operatorname{Pr}\left[\gamma_{2}>\theta_{2, t}, \gamma_{1 \rightarrow 2}<\theta_{2, s}\right] .
\end{aligned}
$$

where $\theta_{1, t}=2^{2 R_{1, t}}-1, \theta_{2, t}=2^{2 R_{2, t}}-1, \theta_{1, s}=$ $2^{2\left(R_{1, t}-R_{1, s}\right)}-1$, and $\theta_{2, s}=2^{2\left(R_{2, t}-R_{2, s}\right)}-1$.

By substituting (2a) and (4) into (6a), we can derive that

$$
\begin{gathered}
\eta_{1}=R_{1, s} \operatorname{Pr}\left[\left(a_{1}-a_{2} \theta_{1, t}\right) \rho_{s}\left|h_{1}\right|^{2}>\left(\rho_{I}+1\right) \theta_{1, t},\right. \\
\left.\frac{a_{1}-a_{2} \theta_{1, s}}{\theta_{1, s}}<\frac{\rho_{s}\left|h_{1}\right|^{2}+\rho_{R}\left|h_{12}\right|^{2}+\rho_{I}+1}{\rho_{s} \rho_{R}\left|h_{1}\right|^{2}\left|h_{12}\right|^{2}}\right] .
\end{gathered}
$$

Clearly, when $a_{1}-a_{2} \theta_{1, t}<0, \eta_{1}=0$. Since $\theta_{1, t} \geq \theta_{1, s}$, on the condition that $a_{1}-a_{2} \theta_{1, t}>0$, (7) can be rewritten as

$\eta_{1}=R_{1, s} \operatorname{Pr}\left[\rho_{s}\left|h_{1}\right|^{2}>\chi_{1}, \frac{\rho_{s} \rho_{R}\left|h_{1}\right|^{2}\left|h_{12}\right|^{2}}{\rho_{s}\left|h_{1}\right|^{2}+\rho_{R}\left|h_{12}\right|^{2}+\rho_{I}+1}<\chi_{2}\right]$,

where $\chi_{1}=\frac{\left(\rho_{I}+1\right) \theta_{1, t}}{a_{1}-a_{2} \theta_{1, t}}$, and $\chi_{2}=\frac{\theta_{1, s}}{a_{1}-a_{2} \theta_{1, s}}$.

To investigate the security of cooperative NOMA with jamming signals, the following theorem is presented to provide an approximation for $\eta_{1}$.

Theorem 1: An approximation of $\eta_{1}$ is given as (9), shown at the top of the next page, where $P_{1}$ is given in (10), $\lambda_{1, l}=\frac{\frac{\left(\rho_{I}+1\right) \theta_{1, t}}{a_{1}-a_{2} \theta_{1, t}}+\frac{\theta_{1, s}}{a_{1}-a_{2} \theta_{1, s}}}{2}+\frac{\frac{\left(\rho_{I}+1\right) \theta_{1, t}}{a_{1}-a_{2} \theta_{1, t}}-\frac{\theta_{1, s}}{a_{1}-a_{2} \theta_{1, s}}}{2} \delta_{l}, \quad \delta_{l}=$ $\cos \left(\frac{(2 l-1) \pi}{2 L}\right)$, and $U(x)=\left\{\begin{array}{ll}1, & x>0 \\ 0 . & x \leq 0\end{array}\right.$.

Proof: By denoting $X=\rho_{s}\left|h_{1}\right|^{2}$ and $Y=\rho_{R}\left|h_{12}\right|^{2}$ with $E[X]=\Omega_{X}$ and $E[Y]=\Omega_{Y}$, and fading parameters $m_{X}$ and $m_{Y}$, the probability term in $\eta_{1}$ can be expressed as

$$
\begin{aligned}
P & =\operatorname{Pr}\left[X>\chi_{1}, \frac{X Y}{X+Y+\rho_{I}+1}<\chi_{2}\right] \\
& =\operatorname{Pr}\left[X>\chi_{1},\left(X-\chi_{2}\right) Y<\chi_{2}\left(X+\rho_{I}+1\right)\right] .
\end{aligned}
$$

Based on the values of $\chi_{1}$ and $\chi_{2}$, two cases are considered:

Case 1: $\chi_{1} \leq \chi_{2}$; Case 2: $\chi_{1}>\chi_{2}$.

For Case 1 , the above probability can be expressed as

$$
P_{1}=\int_{\chi_{1}}^{\chi_{2}} f_{X}(x) d x+\int_{\chi_{2}}^{\infty} \int_{0}^{\frac{\chi_{2}\left(x+\rho_{I}+1\right)}{x-\chi_{2}}} f_{Y}(y) f_{X}(x) d y d x .
$$

By inserting the cumulative distribution function (CDF) $F_{X}(x)=1-\sum_{r=0}^{m_{X}-1}\left(\frac{m_{X} x}{\Omega_{X}}\right)^{r} \frac{1}{r !} e^{-\frac{m_{X} x}{\Omega_{X}}}$ and probability density function (PDF) $f_{Y}(y)=\left(\frac{m_{Y}}{\Omega_{Y}}\right)^{m_{Y}} \frac{y^{m_{Y}-1}}{\Gamma\left(m_{Y}\right)} e^{-\frac{m_{Y} y}{\Omega_{Y}}}$, (12) can be rewritten as

$P_{1}=\sum_{r=0}^{m_{X}-1}\left(\frac{m_{X} \chi_{1}}{\Omega_{X}}\right)^{r} \frac{1}{r !} e^{-\frac{m_{X} \chi_{1}}{\Omega_{X}}}-\sum_{r=0}^{m_{Y}-1}\left(\frac{m_{X}}{\Omega_{X}}\right)^{m_{X}} \frac{1}{r !} \frac{1}{\Gamma\left(m_{X}\right)}$ 


$$
\begin{aligned}
\eta_{1} & =U\left(a_{1}-a_{2} \theta_{1, t}\right) R_{1, s}\left(U\left(\chi_{2}-\chi_{1}\right) P_{1}+U\left(\chi_{1}-\chi_{2}\right)\left(P_{1}-\sum_{l=1}^{L} \sum_{r=0}^{m_{12}-1}\left(\frac{m_{1}}{\Omega_{1}}\right)^{m_{1}} \frac{1}{r !} \frac{1}{\Gamma\left(m_{1}\right)} \omega_{l} \sqrt{\left(\lambda_{1, l}-\chi_{2}\right)\left(\chi_{1}-\lambda_{1, l}\right)}\right.\right. \\
& \left.\times\left(\frac{m_{12} \chi_{2}\left(\lambda_{1, l}+\rho_{I}+1\right)}{\Omega_{12}\left(\lambda_{1, l}-\chi_{2}\right)}\right)^{r} e^{-\left(\frac{m_{12} \chi_{2}\left(\lambda_{1, l}+\rho_{I}+1\right)}{\Omega_{12}\left(\lambda_{1, l}-\chi_{2}\right)}+\frac{m_{1} \lambda_{1, l}}{\Omega_{1}}\right)} \lambda_{1, l}^{m_{1}-1}\right),
\end{aligned}
$$

where $P_{1}=\sum_{r=0}^{m_{1}-1}\left(\frac{m_{1} \chi_{1}}{\rho_{s} \Omega_{1}}\right)^{r} \frac{1}{r !} e^{-\frac{m_{1} \chi_{1}}{\rho_{s} \Omega_{1}}}-\sum_{r=0}^{m_{12}-1} \sum_{s=0}^{r} \sum_{t=0}^{m_{1}-1}\left(\begin{array}{c}r \\ s\end{array}\right)\left(\begin{array}{c}m_{1}-1 \\ t\end{array}\right)\left(\frac{m_{1}}{\rho_{s} \Omega_{1}}\right)^{m_{1}}\left(\frac{m_{12} \chi_{2}}{\rho_{R} \Omega_{12}}\right)^{r} e^{-\chi_{2}\left(\frac{m_{1}}{\rho_{s} \Omega_{1}}+\frac{m_{12}}{\rho_{R} \Omega_{12}}\right)}$

$$
\times \frac{1}{r !}\left(\chi_{2}+\rho_{I}+1\right)^{s} \chi_{2}^{m_{1}-1-t} \frac{2}{\Gamma\left(m_{1}\right)} K_{t-s+1} 2 \sqrt{\frac{m_{1} m_{12} \chi_{2}\left(\chi_{2}+\rho_{I}+1\right)}{\rho_{s} \rho_{R} \Omega_{1} \Omega_{12}}}\left(\frac{m_{12} \rho_{s} \Omega_{1} \chi_{2}\left(\chi_{2}+\rho_{I}+1\right)}{m_{1} \rho_{R} \Omega_{12}}\right)^{\frac{t-s+1}{2}},
$$

$$
\begin{aligned}
\eta_{2} & =U\left(\theta_{2, s}\left(\rho_{I}+1\right)-\theta_{2, t}\right) R_{2, s} \sum_{l=1}^{L} \sum_{r=0}^{m_{12}-1}\left(\frac{m_{1}}{\rho_{s} \Omega_{1}}\right)^{m_{1}} \frac{1}{\Gamma\left(m_{1}\right)} \frac{1}{r !} \omega_{l} \sqrt{\left(\lambda_{2, l}-\frac{\theta_{2, t}}{a_{2}}\right)\left(\frac{\theta_{2, s}\left(\rho_{I}+1\right)}{a_{2}}-\lambda_{2, l}\right)\left(\frac{m_{12} \phi\left(\lambda_{2, l}\right)}{\rho_{R} \Omega_{12}}\right)^{r} \lambda_{2, l}^{m_{1}-1}} \\
& \times e^{-\left(\frac{m_{1} \lambda_{2, l}}{\rho_{s} \Omega_{1}}+\frac{m_{12} \phi\left(\lambda_{2, l}\right)}{\rho_{R} \Omega_{12}}\right)}
\end{aligned}
$$

$$
\begin{aligned}
& \times \int_{\chi_{2}}^{\infty}\left(\frac{m_{Y} \chi_{2}\left(x+\rho_{I}+1\right)}{\Omega_{Y}\left(x-\chi_{2}\right)}\right)^{r} e^{-\frac{m_{Y} \chi_{2}\left(x+\rho_{I}+1\right)}{\Omega_{Y}\left(x-\chi_{2}\right)}} x^{m_{X}-1} e^{-\frac{m_{X} x}{\Omega_{X}}} d x \\
& \stackrel{a}{=} P_{1}^{1}-\sum_{r=0}^{m_{Y}-1} \sum_{s=0}^{r} \sum_{t=0}^{m_{X}-1}\left(\begin{array}{c}
r \\
s
\end{array}\right)\left(\begin{array}{c}
m_{X}-1 \\
t
\end{array}\right)\left(\frac{m_{X}}{\Omega_{X}}\right)^{m_{X}} \frac{1}{r !}\left(\frac{m_{Y} \chi_{2}}{\Omega_{Y}}\right)^{r} \\
& \times e^{-\chi_{2}\left(\frac{m_{X}}{\Omega_{X}}+\frac{m_{Y}}{\Omega_{Y}}\right)}\left(\chi_{2}+\rho_{I}+1\right)^{s} \chi_{2}^{m_{X}-1-t} \frac{1}{\Gamma\left(m_{X}\right)} \\
& \times \int_{\chi_{2}}^{\infty}\left(x-\chi_{2}\right)^{t-s} e^{-\left(\frac{m_{X}\left(x-\chi_{2}\right)}{\Omega_{X}}+\frac{m_{Y} \chi_{2}\left(\chi_{2}+\rho_{I}+1\right)}{\Omega_{Y}\left(x-\chi_{2}\right)}\right)} d x \\
& \stackrel{b}{=} P_{1}^{1}-\sum_{r=0}^{m_{Y}-1} \sum_{s=0}^{r} \sum_{t=0}^{m_{X}-1}\left(\begin{array}{c}
r \\
s
\end{array}\right)\left(\begin{array}{c}
m_{X}-1 \\
t
\end{array}\right)\left(\frac{m_{X}}{\Omega_{X}}\right)^{m_{X}} \frac{1}{r !}\left(\frac{m_{Y} \chi_{2}}{\Omega_{Y}}\right)^{r} \\
& \times e^{-\chi_{2}\left(\frac{m_{X}}{\Omega_{X}}+\frac{m_{Y}}{\Omega_{Y}}\right)\left(\chi_{2}+\rho_{I}+1\right)^{s} \chi_{2}^{m_{X}-1-t} \frac{2}{\Gamma\left(m_{X}\right)}} \\
& \times K_{t-s+1}\left(2 \sqrt{\frac{m_{X} m_{Y} \chi_{2}\left(\chi_{2}+\rho_{I}+1\right)}{\Omega_{X} \Omega_{Y}}}\right) \\
& \times\left(\frac{m_{Y} \Omega_{X} \chi_{2}\left(\chi_{2}+\rho_{I}+1\right)}{m_{X} \Omega_{Y}}\right)^{\frac{t-s+1}{2}},
\end{aligned}
$$

where (a) is derived by denoting $P_{1}^{1} \triangleq$ $\sum_{r=0}^{m_{X}-1}\left(\frac{m_{X} \chi_{1}}{\Omega_{X}}\right)^{r} \frac{1}{r !} e^{-\frac{m_{X} \chi_{1}}{\Omega_{X}}}$, (b) is obtained by adopting [16, Eq. $(3.478,4)$ ], and $K_{v}(z)$ represents the Bessel functions of imaginary argument [17].

For Case 2, the probability in (11) can be reformulated as

$$
\begin{aligned}
P_{2} & =\int_{\chi_{1}}^{\infty} \int_{0}^{\frac{\chi_{2}\left(x+\rho_{I}+1\right)}{x-\chi_{2}}} f_{Y}(y) f_{X}(x) d y d x \\
& =P_{1}+\int_{\chi_{2}}^{x_{1}} \int_{0}^{\frac{\chi_{2}\left(x+\rho_{I}+1\right)}{x-\chi_{2}}} f_{Y}(y) f_{X}(x) d y d x .
\end{aligned}
$$

By applying Gauss-Chebyshev integration, the approximation for $P_{2}$ can be given by

$$
P_{2}=P_{1}+\sum_{l=1}^{L} \sum_{r=0}^{m_{Y}-1}\left(\frac{m_{X}}{\Omega_{X}}\right)^{m_{X}} \frac{1}{r !} \frac{1}{\Gamma\left(m_{X}\right)} \omega_{l} \lambda(l)^{m_{X}-1}
$$

$$
\begin{aligned}
& \times \sqrt{\left(\lambda_{l}-\chi_{2}\right)\left(\chi_{1}-\lambda_{l}\right)}\left(\frac{m_{Y} \chi_{2}\left(y(l)+\rho_{I}+1\right)}{\Omega_{Y}\left(y(l)-\chi_{2}\right)}\right)^{r} \\
& \times e^{-\left(\frac{m_{Y} \chi_{2}\left(y(l)+\rho_{I}+1\right)}{\Omega_{Y}\left(y(l)-\chi_{2}\right)}+\frac{m_{X} y(l)}{\Omega_{X}}\right)},
\end{aligned}
$$

where $\omega_{l}=\frac{\pi}{L}, \quad L$ denotes the approximation order for Gauss-Chebyshev integration, $\lambda_{l}=\frac{\chi_{1}+\chi_{2}}{2}+\frac{\chi_{1}-\chi_{2}}{2} \delta_{l}$, and $\delta_{l}=\cos \left(\frac{(2 l-1) \pi}{2 L}\right)$.

Hence, a complete analytical expression for the probability term in $\eta_{1}$ can be written as $P=U\left(\chi_{2}-\chi_{1}\right) P_{1}+U\left(\chi_{1}-\right.$ $\left.\chi_{2}\right) P_{2}$. Then, (9) can be derived via replacing $\Omega_{X}$ and $\Omega_{Y}$ with $\rho_{s} \Omega_{1}$ and $\rho_{R} \Omega_{12}$, respectively.

Remark 1: According to (9), $\eta_{1}$ is a monotonically decreasing function of $P_{R}$ and $P_{I}$, but increases monotonically with $P_{s}$. Moreover, the power allocation factors and the code-word rate need to be carefully set to satisfy $a_{1}-a_{2 \theta_{1, t}}>0$ to achieve nonzero $\eta_{1}$.

For $\eta_{2}$, by substituting (2b) and (5) into (6b), we have

$$
\eta_{2}=R_{2, s} \operatorname{Pr}\left[\frac{\rho_{s} \rho_{R}\left|h_{1}\right|^{2}\left|h_{12}\right|^{2}}{\rho_{s}\left|h_{1}\right|^{2}+\rho_{R}\left|h_{12}\right|^{2}+\rho_{I}+1}>z_{1}, \rho_{s}\left|h_{1}\right|^{2}<z_{2}\right],
$$

where $z_{1}=\frac{\theta_{2, t}}{a_{2}}$ and $z_{2}=\frac{\theta_{2, s}\left(\rho_{I}+1\right)}{a_{2}}$.

Theorem 2: An exact expression for $\eta_{2}$ is given in (17), shown at the top of this page, where $\lambda_{2, l}=$ $\frac{\frac{\theta_{2, t}}{a_{2}}+\frac{\theta_{2, s}\left(\rho_{I}+1\right)}{a_{2}}}{2}+\frac{\frac{\theta_{2, t}}{a_{2}}-\frac{\theta_{2, s}\left(\rho_{I}+1\right)}{a_{2}}}{2} \delta_{l}, \delta_{l}=\cos \left(\frac{(2 l-1) \pi}{2 L}\right)$, and $\phi(x)=\frac{\theta_{2, t}\left(x+\rho_{I}+1\right)}{a_{2} x-\theta_{2, t}}$.

Proof: By denoting $X=\rho_{s}\left|h_{1}\right|^{2}$ and $Y=\rho_{R}\left|h_{12}\right|^{2}$, the probability term in $\eta_{2}$ can be written as

$$
\begin{aligned}
P & =\operatorname{Pr}\left[X<z_{2}, \frac{X Y}{X+Y+\rho_{I}+1}>z_{1}\right] \\
& =\sum_{r=0}^{m_{Y}-1}\left(\frac{m_{X}}{\Omega_{X}}\right)^{m_{X}} \frac{1}{\Gamma\left(m_{X}\right)} \frac{1}{r !} \int_{z_{1}}^{z_{2}}\left(\frac{m_{Y} \phi(x)}{\Omega_{Y}}\right)^{r} x^{m_{X}-1} \\
& \times e^{-\left(\frac{m_{X} x}{\Omega_{X}}+\frac{m_{Y} \phi(x)}{\Omega_{Y}}\right)} d x
\end{aligned}
$$




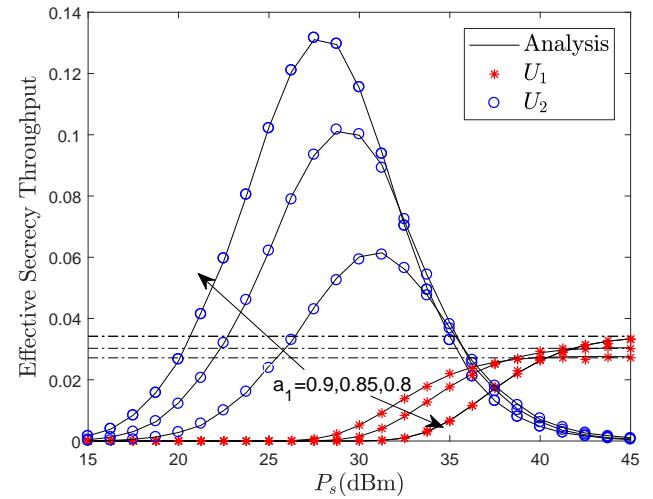

Fig. 1. The ESTs of $U_{1}$ and $U_{2}$ vs. $P_{s}$ with $P_{R}=-2 \mathrm{dBm}$ and $P_{I}=-10 \mathrm{dBm}$.

where $\phi(x)=\frac{z_{1}\left(x+\rho_{I}+1\right)}{x-z_{1}}$.

By employing Gauss-Chebyshev integration, the approximation for (18) is derived as

$$
\begin{aligned}
& P=\sum_{l=1}^{L} \sum_{r=0}^{m_{Y}-1}\left(\frac{m_{X}}{\Omega_{X}}\right)^{m_{X}} \frac{1}{\Gamma\left(m_{X}\right)} \frac{1}{r !} \omega_{l} \sqrt{\left(\lambda_{l}-z_{1}\right)\left(z_{2}-\lambda_{l}\right)} \\
& \times\left(\frac{m_{Y} \phi\left(\lambda_{l}\right)}{\Omega_{Y}}\right)^{r} y_{l}^{m_{X}-1} e^{-\left(\frac{m_{X} y_{l}}{\Omega_{X}}+\frac{m_{Y} \phi\left(y_{l}\right)}{\Omega_{Y}}\right)}
\end{aligned}
$$

where $\lambda_{l}=\frac{z_{1}+z_{2}}{2}+\frac{z_{1}-z_{2}}{2} \delta_{l}$, and $\delta_{l}=\cos \left(\frac{(2 l-1) \pi}{2 L}\right)$.

By replacing $\Omega_{X}$ and $\Omega_{Y}$ with $\rho_{s} \Omega_{1}$ and $\rho_{R} \Omega_{12}$, and after some algebraic manipulations, an approximation for $\eta_{2}$ can be finally given in (17).

Remark 2: (17) indicates that $\eta_{2}$ is not a monotonic function of either $P_{s}$ or $P_{I}$, thus $P_{s}$ and $P_{I}$ should be carefully chosen. Particularly, a nonzero $\eta_{2}$ can only be obtained under the condition that $\rho_{I}>\frac{\theta_{2, t}}{\theta_{2, s}}-1$, which shows that jamming is essential for the secure transmission of $U_{2}$. Note that an infinite $P_{I}$ results in zero $\eta_{2}$, which reveals that $\eta_{2}$ can be maximized with an optimal $P_{I}$.

\section{B. Asymptotic Expressions}

Based on the derived expressions for $\eta_{1}$ and $\eta_{2}$, we can see that the infinite $P_{R}$ and $P_{I}$ result in zero $\eta_{1}$, and $\eta_{2}$ becomes zero with infinite $P_{s}$. Thus, we provide an asymptotic expression for $\eta_{1}$ as $P_{s} \rightarrow \infty$, and one for $\eta_{2}$ as $P_{R} \rightarrow \infty$.

When $P_{s} \rightarrow \infty$ and $a_{1}-a_{2} \theta_{1, t}>0, \eta_{1}$ can be expressed as

$$
\eta_{1}^{P_{s} \rightarrow \infty}=R_{1, s}\left[\rho_{R}\left|h_{12}\right|^{2}<\frac{\theta_{1, s}}{a_{1}-a_{2} \theta_{1, s}}\right] .
$$

By employing the CDF $F_{X}(x)=1-$ $\sum_{r=0}^{m_{X}-1}\left(\frac{m_{X} x}{\Omega_{X}}\right)^{r} \frac{1}{r !} e^{-\frac{m_{X} x}{\Omega_{X}}}$, we can derive an asymptotic closed-form expression for $\eta_{1}$ as

$$
\begin{aligned}
\eta_{1}^{P_{s} \rightarrow \infty} & =R_{1, s}\left(1-\sum_{r=0}^{m_{12}-1}\left(\frac{m_{12} \theta_{1, s}}{\left(a_{1}-a_{2} \theta_{1, s}\right) \rho_{R} \Omega_{12}}\right)^{r} \frac{1}{r !}\right. \\
& \left.\times e^{-\frac{m_{12} \theta_{1, s}}{\left(a_{1}-a_{2} \theta_{1, s}\right) \rho_{R} \Omega_{12}}}\right) .
\end{aligned}
$$

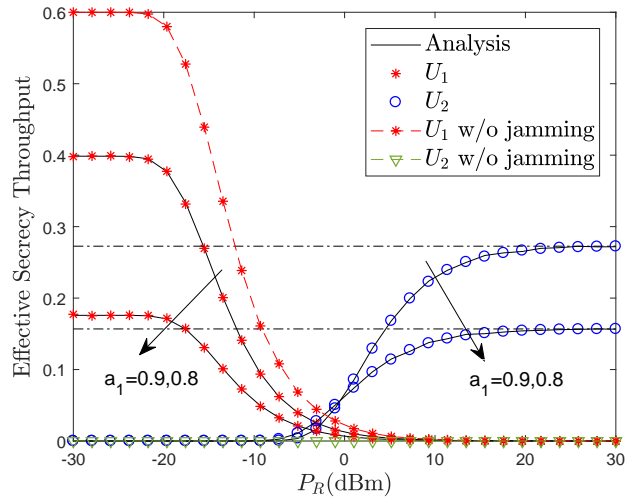

Fig. 2. The ESTs of $U_{1}$ and $U_{2}$ vs. $P_{R}$ with $P_{s}=36 \mathrm{dBm}$ and $P_{I}=-10 \mathrm{dBm}$.

When $P_{R} \rightarrow \infty$, an asymptotic closed-form expression for $\eta_{2}$ can be written as

$$
\begin{aligned}
& \eta_{2}^{P_{R} \rightarrow \infty}=R_{2, s}\left(\sum_{r=0}^{m_{1}-1}\left(\frac{m_{1} \theta_{2, t}}{a_{2} \rho_{s} \Omega_{1}}\right)^{r} \frac{1}{r !} e^{-\frac{m_{1} \theta_{2, t}}{a_{2} \rho_{s} \Omega_{1}}}\right. \\
& \left.-\sum_{r=0}^{m_{1}-1}\left(\frac{m_{1} \theta_{2, s}\left(\rho_{I}+1\right)}{a_{2} \rho_{s} \Omega_{1}}\right)^{r} \frac{1}{r !} e^{-\frac{m_{1} \theta_{2, s}\left(\rho_{I}+1\right)}{a_{2} \rho_{s} \Omega_{1}}}\right) .
\end{aligned}
$$

Remark 3: According to (21) and (22), we can see that the ESTs of $U_{1}$ and $U_{2}$ tend to nonzero constants when $P_{s} \rightarrow \infty$ and $P_{R} \rightarrow \infty$, respectively, as $\eta_{1}^{P_{s} \rightarrow \infty}$ is determined by the second slot, while $\eta_{2}^{P_{R} \rightarrow \infty}$ is determined by the first slot.

\section{Numerical Results}

In this section, we present numerical results to evaluate the performance of the proposed cooperative NOMA system. The parameters are set as follows, unless otherwise stated. The average channel gains are chosen as $\Omega_{i}=\frac{1}{d_{i}^{\alpha}}$, where $i \in\{1,12\}$, the distance $d_{i}=20 \mathrm{~m}$, and $\alpha=2.7^{2}$ denoting the path loss exponent. Without loss of generality, we set $L=50, R_{1, t}=R_{2, t}=1 \mathrm{BPCU}, R_{1, s}=R_{2, s}=0.6$ BPCU, and uniform Nakagami- $m$ fading is assumed for all channels, where $m_{1}=m_{12}=2$. The background noise power is assumed to be $-50 \mathrm{dBm}$.

In Fig. 1, the relationship between the EST and the transmit power at the BS $\left(P_{s}\right)$ is presented. As can be observed from Fig. 1, the derived analytical results are consistent with those from the simulations, and both users can achieve positive secrecy rate when $P_{s}$ ranges from $33 \mathrm{dBm}$ to $42 \mathrm{dBm}$. We can see that increasing $P_{s}$ enhances the secrecy performance of $U_{1}$, and the EST of $U_{1}$ converges to asymptotic values as $P_{s}$ gradually increases. Moreover, the EST of $U_{2}$ first increases and then decreases, which reveals that there exists an optimal $P_{s}$ to maximize the EST of $U_{2}$. In addition, for the near user $U_{1}$, decreasing the power allocation fraction at the near user results in better secrecy performance in the low transmit power regime, while better system performance can be achieved when a larger power is allocated to the near user in the high transmit power region. However, the relationship between the far user $U_{2}$ and the power coefficient $a_{1}$ is inverse to that of $U_{1}$ and $a_{1}$. The reason for this is that, the impact of the jamming signal is negligible when $P_{s}$ becomes larger. 

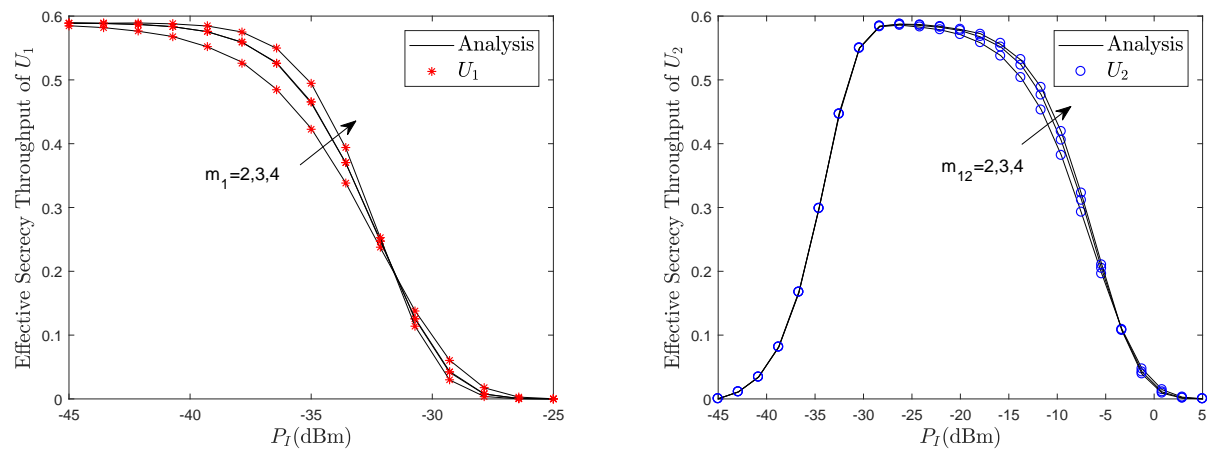

(a) The EST of $U_{1}$ vs. $P_{I}$ with $P_{s}=10 \mathrm{dBm}$ and $P_{R}=$ (b) The EST of $U_{2}$ vs. $P_{I}$ with $P_{s}=10 \mathrm{dBm}$ and $-20 \mathrm{dBm}$

$P_{R}=20 \mathrm{dBm}$

Fig. 3. The ESTs vs. $P_{I}$.

Fig. 2 plots the ESTs of $U_{1}$ and $U_{2}$ versus $P_{R}$ with $P_{s}=36$ $\mathrm{dBm}$ and $P_{I}=-10 \mathrm{dBm}$. Cooperative NOMA without jamming, namely ' $U_{1}$ w/o jamming' and ' $U_{2}$ w/o jamming', is plotted as the comparison scheme. For cooperative NOMA without jamming, we can see that though the EST of $U_{1}$ outperforms that of the proposed jamming strategy, the EST of $U_{2}$ remains at zero, thus indicating that $U_{2}$ cannot achieve secure transmission. The derived analytical results coincide precisely with those of the simulations. We can see that the EST of $U_{1}$ decreases with $P_{R}$, and the EST of $U_{2}$ converges to asymptotic values as $P_{R}$ increases. This is because increasing $P_{R}$ results in a larger achievable rate of $U_{2}$ as well as a larger interception rate for $U_{2}$ detecting the information of $U_{1}$. Moreover, $U_{1}$ can always benefit from the larger power allocated to the near user, while increasing $a_{1}$ degrades the secrecy performance of $U_{2}$ in the low $P_{R}$ region, but improves the EST of $U_{2}$ when $P_{R}$ becomes larger. The reason for this is that, for the data rate of $U_{2}$, the impact of the power allocation factor $a_{2}$ overwhelms the effect of $P_{R}$ when $P_{R}$ is relatively small, and $P_{R}$ plays the leading role in the high power region.

In Fig. 3, the ESTs of $U_{1}$ and $U_{2}$ versus $P_{I}$ are presented, respectively. As can be seen from Fig. 3. (a), the EST of $U_{1}$ decreases with $P_{I}$ since $P_{I}$ interferes the achievable SINR of $U_{1}$ as well as the intercept SINR of $U_{2}$. The EST of $U_{2}$ first increases and then decreases, thus an optimal $P_{I}$ exists to maximize the EST. Moreover, for $U_{1}$ and $U_{2}$, more stable channels, i.e., larger Nakagami- $m$ fading parameters, contribute to the improvement of the EST in the low power regime, and degrade the secrecy performance with a higher $P_{I}$.

\section{CONCLUSION}

In this letter, to address open security issues for a twouser cooperative NOMA network, the inverse power allocation and SIC decoding order as well as a novel jamming strategy have been proposed to overcome information leakage in the presence of internal untrusted near and far users. To evaluate the performance of the proposed system, Gauss-Chebyshev approximations and an asymptotic analysis of the ESTs have been derived for both users. Numerical results have validated that the proposed jamming strategy supports secure transmissions, while the jamming power needs to be chosen carefully to achieve satisfactory secrecy performance. The impacts of $P_{s}$ and $P_{R}$ on EST values have also been illustrated.

\section{REFERENCES}

[1] Z. Ding, Z. Yang, P. Fan, and H. V. Poor. On the performance of nonorthogonal multiple access in $5 \mathrm{G}$ systems with randomly deployed users. IEEE Signal. Proc. Lett., vol. 21, no. 12, pp. 1501-1505, July 2014.

[2] Y. Liu, Z. Ding, M. Elkashlan, and H. V. Poor. Cooperative nonorthogonal multiple access with simultaneous wireless information and power transfer. IEEE J. Sel. Areas Commun., vol. 34, no. 4, pp. 938-953, Apr. 2016.

[3] L. Lv, H. Jiang, Z. Ding, Q. Ye, N. Al-Dhahir, and J. Chen. Secure non-orthogonal multiple access: An interference engineering perspective. IEEE Network, 2020, early access.

[4] W. Yu, A. Chorti, L. Musavian, H. V. Poor, and Q. Ni. Effective secrecy rate for a downlink NOMA network. IEEE Trans. Wireless Commun., vol. 18 , no. 12 , pp. 5673-5690, Dec. 2019.

[5] R. Ruby, T. Riihonen, K. Wu, Y. Liu, and B. M. ElHalawany. Performance analysis of multi-phase cooperative NOMA systems under passive eavesdropping. Signal Processing, 182:107934, 2021.

[6] J. Chen, L. Yang, and M.-S Alouini. Physical layer security for cooperative NOMA systems. IEEE Trans. Veh. Technol., 67(5):46454649, May 2018.

[7] B. M. ElHalawany, R. Ruby, T. Riihonen, and K. Wu. Performance of cooperative NOMA systems under passive eavesdropping. In Proc. IEEE Global Commun. Conf. (GLOBECOM), pages 1-6. IEEE, Abu Dhabi, United Arab Emirates, Dec. 2018.

[8] B. M. ElHalawany and K. Wu. Physical-layer security of NOMA systems under untrusted users. In Proc. IEEE GLOBECOM, United Arab Emirates, pp. 1-6, Dec. 2018.

[9] K. Cao, B. Wang, H. Ding, T. Li, J. Tian, and F. Gong. Secure transmission designs for NOMA systems against internal and external eavesdropping. IEEE Trans. Inf. Forensics Security, vol. 15, pp. 29302953, Mar. 2020.

[10] C. Zhang, F. Jia, Z. Zhang, J. Ge, and F. Gong. Physical layer security designs for 5G NOMA systems with a stronger near-end internal eavesdropper. IEEE Trans. Veh. Technol., vol. 69, no. 11, pp. 1300513017, Nov. 2020.

[11] S. Thapar, D. Mishra, and R. Saini. Novel outage-aware NOMA protocol for secrecy fairness maximization among untrusted users. IEEE Trans. Veh. Technol., vol. 69, no. 11, pp. 13259-13272, Sep. 2020.

[12] Z. Xiang, W. Yang, G. Pan, Y. Cai, and X. Sun. Secure transmission in non-orthogonal multiple access networks with an untrusted relay. IEEE Wireless Commun. Lett., vol. 8, no. 3, pp. 905-908, June 2019.

[13] C. Yu, HL. Ko, X. Peng, X. Xie, and P. Zhu. Jammer-aided secure communications for cooperative NOMA systems. IEEE Commun. Lett., vol. 23, no. 11, pp. 1935-1939, Nov. 2019.

[14] A. D. Wyner. The wire-tap channel. Bell Syst. Tech. J., vol. 54, no. 8, pp. 1355-1387, Oct. 1975.

[15] A. Chorti, C. Hollanti, JC. Belfiore, and H. V. Poor. Physical layer security: A paradigm shift in data confidentiality. Lecture Notes in Electrical Engineering. Springer Verlag, Germany, 2016.

[16] I. S. Gradshteyn and I. M. Ryzhik. Table of Integrals, series and products. 7th ed. New York, NY, USA: Academic, 2007.

[17] G. Dattoli and A. Torre. Theory and applications of generalized Bessel functions. Rome, Italy: Aracne Editrice, 1996. 